\title{
Review of: "Vanucizumab mode of action: Serial biomarkers in plasma, tumor, and skin-wound- healing biopsies"
}

\author{
Chen Wang, Yongmin Yan ${ }^{1}$ \\ 1 Jiangsu University
}

Potential competing interests: The author(s) declared that no potential competing interests exist.

Tumor-associated angiogenesis is a highly complex process resulting from the imbalance relationship between pro-angiogenic and antiangiogenic factors. Angiogenesis is accompanied by the whole process of tumor growth, metastasis, and even drug resistance [1]. Targeted therapy against tumor angiogenesis has become an effective treatment strategy. Cancer cells release pro-angiogenic factors to promote the continuous formation of new blood vessels in the tumor. Previous studies have found more than 40 crucial angiogenic factors, in which VEGF/VEGFR and Ang-2/Tie-2 (receptor) play a central role during the progress. VEGF is the mitogen with the highest specificity targeting endothelial cells and one of the most influential factors in angiogenesis that directly stimulate the movement, proliferation, and division of vascular endothelial cells. The humanized anti-VEGF monoclonal antibody Bevacizumab is the first drug approved by the FDA to inhibit tumor angiogenesis [2]. Another factor, Angiopoietin-2 (Ang-2), is the ligand of tyrosine kinase (Tie-2) receptor, which is secreted by endothelial cells and stored in the Weibel-Palade body (WPB) [3]. Both VEGF and ANG-2 are critical regulators of tumor angiogenesis, and their activity is often abnormally increased in tumor angiogenesis. Bispecific antibody approaches that simultaneously block signal transduction triggered by VEGF and ANG-2 increase tumor-targeting specificity. Vanucizumab is a bi-specific IgG1-like monoclonal antibody using CrossMab technology that simultaneously blocks VEGFA and Ang-2 from interacting with their receptors [4]. At present, the anti-tumor angiogenesis effect of vanucizumab in treating solid clinical tumors is still unclear.

In another phase I clinical study from the same group, Florian et al. and his colleagues evaluated the safety, tolerability, pharmacokinetics, and pharmacodynamics of vanucizumab in patients with advanced solid tumors (NCT01688206) [5]. I read this paper written by Florian et al. with great interest. As part of phase II clinical study, Florian et al. evaluated the pharmacodynamic effects of vanucizumab in 32 patients. To assess the anti-tumor angiogenesis during the treatments, the author examined the level of vascularrelated biomarkers VEGF-A and Ang-2 in the plasma of tumor patients. The reductions of circulating unbound VEGF-A and Ang-2 concentrations indicate the efficacy of this vanucizumab treatment. The authors also described the effect of vanucizumab therapy on the microvessel density (MVD) and density of proliferating vessels in tumor and skin-wound-healing biopsies. Considering tumor biopsies exist many inconveniences in the clinical practices. Therefore, the authors conducted skin-wound-healing biopsies 
simultaneously to analyze the expression of vascular markers after vanucizumab therapy. They explored whether skin biopsies could be a surrogate means instead of tumor biopsies to determine the intra-tumoral pharmacodynamics of vanucizumab. The MVD and vascular proliferation results of skin wound healing biopsies are similar to tumor biopsy results. Florian et al. proposed for the first time to explore the intratumoral pharmacodynamic effect of an investigational new antiangiogenic antibody by assessing paired tumor- and corresponding skin biopsies. Vanucizumab may effectively reduce vascular-related biomarkers in plasma, tumor samples, and wound-healing biopsies (Figure 1).

Antiangiogenic treatments contribute to improving the integrity of the tumor vasculature and increase the associated T-cell infiltration. Gene expression analysis of tumor tissues showed increased expression of pro-inflammatory factors, suggesting that vanucizumab may recruit and potentially activate lymphocytes. Evaluation of immune markers for patients with paired tumor samples classified also showed that vanucizumab $(30 \mathrm{mg} / \mathrm{kg}$ ) treatment indued a low CD8 + T-cell infiltration levels (Figure 1). However, no significant CT8 cell infiltration was observed in the low-dose group $(30 \mathrm{mg} / \mathrm{kg})$. Larger samples sizes are needed to illustrate this issue further. The authors carefully controlled the selection of patients with advanced solid tumors, including colon/rectum, breast, lung, stomach/esophagus, pancreas, cervix, kidney, skin, thyroid, and uterus. Vanucizumab revealed a consistent biological effect for vascular markers in the selected patients. Solid tumors may comprise subpopulations of cells with distinct genomic alterations within the same tumor. There is increasing evidence that anti-angiogenesis therapy for patients with advanced solid tumors has apparent heterogeneity [6] Intratumor heterogeneity may contribute to the failure of cancer therapy by initiating phenotypic diversity enabling drug resistance to emerge, and introducing tumor sampling bias. To fully understand the efficacy of vanucizumab, the intratumor heterogeneity of vanucizumab treatment is worthy of further exploration.

Emerging cancer treatments like immune checkpoint inhibitors (ICls), bio-targeted therapy have revolutionized clinical practice in recent years [7]. However, the real breakthrough in the treatment of advanced solid tumors still faces numerous challenges, including the lack of specific antigen recognition on the surface of solid tumors and the suppression of the tumor microenvironment. Therefore, we need a new treatment technology to help us better Fight against solid tumors. Antiangiogenic therapy inhibits angiogenesis, reduces the number of immunosuppressive cells in the tumor microenvironment, and improves tumor-infiltrating lymphocyte (TIL) and cytotoxic T lymphocytes (CTL) to overcome tumor immune escape. The bispecific antibody has a broad prospect of application in clinical treatments in various clinical trials of advanced solid tumors. The study by Florian et al. gave the necessary attention to seek a worthy bispecific antibody for the treatments of advanced solid tumors. Previous clinical investigations indicate that antiangiogenic drugs combined with chemotherapy, radiotherapy, targeted therapy, and immunotherapy may be a mutually beneficial treatment strategy [8-10]. Further use of vanucizumab combined with other treatments may exert greater efficacy in the treatment of solid tumors. In summary, vanucizumab is a promising anti-tumor angiogenesis drug, representing significant progress in understanding and treating advanced solid tumors. Paired tumor and skin-wound-healing biopsies 
provide a new perspective for exploring anti-angiogenesis therapy. Future studies with larger sample sizes are required to observe the pharmacodynamic effect in advanced solid tumors.

\section{References}

[1] R.N. Gacche, R.J. Meshram, Angiogenic factors as potential drug target: efficacy and limitations of antiangiogenic therapy, Biochim Biophys Acta 1846 (2014)161-79.

[2] Y. Ogata, M. Shimokawa, T. Tanaka, et al., A prospective study of XELOX plus bevacizumab as first-line therapy in Japanese patients with metastatic colorectal cancer (KSCC 0902), INT J CLIN ONCOL 21 (2016)335-343.

[3] P.C. Maisonpierre, C. Suri, P.F. Jones, et al., Angiopoietin-2, a natural antagonist for Tie2 that disrupts in vivo angiogenesis, SCIENCE 277 (1997)55-60.

[4] Y. Kienast, C. Klein, W. Scheuer, et al., Ang-2-VEGF-A CrossMab, a novel bispecific human IgG1 antibody blocking VEGF-A and Ang-2 functions simultaneously, mediates potent antitumor, antiangiogenic, and antimetastatic efficacy, CLIN CANCER RES 19 (2013)6730-40.

[5] M. Hidalgo, M. Martinez-Garcia, C. Le Tourneau, et al., First-in-Human Phase I Study of Single-agent Vanucizumab, A First-in-Class Bispecific Anti-Angiopoietin-2/Anti-VEGF-A Antibody, in Adult Patients with Advanced Solid Tumors, CLIN CANCER RES 24 (2018)1536-1545.

[6] T.A. Yap, M. Gerlinger, P.A. Futreal, et al., Intratumor heterogeneity: seeing the wood for the trees, SCl TRANSL MED 4 (2012)127ps10.

[7] M. Hong, J.D. Clubb, Y.Y. Chen, Engineering CAR-T Cells for Next-Generation Cancer Therapy, CANCER CELL 38 (2020)473-488.

[8] L. Tian, A. Goldstein, H. Wang, et al., Mutual regulation of tumour vessel normalization and immunostimulatory reprogramming, NATURE 544 (2017)250-254.

[9] M. de Miguel, E. Calvo, T cell engagers in solid tumors kick the door down, CANCER CELL 39 (2021)1461-1463.

[10] M. Cully, Cancer: Bispecific antibody directs T cells to solid tumours, NAT REV DRUG DISCOV 16 (2017)826-827. 


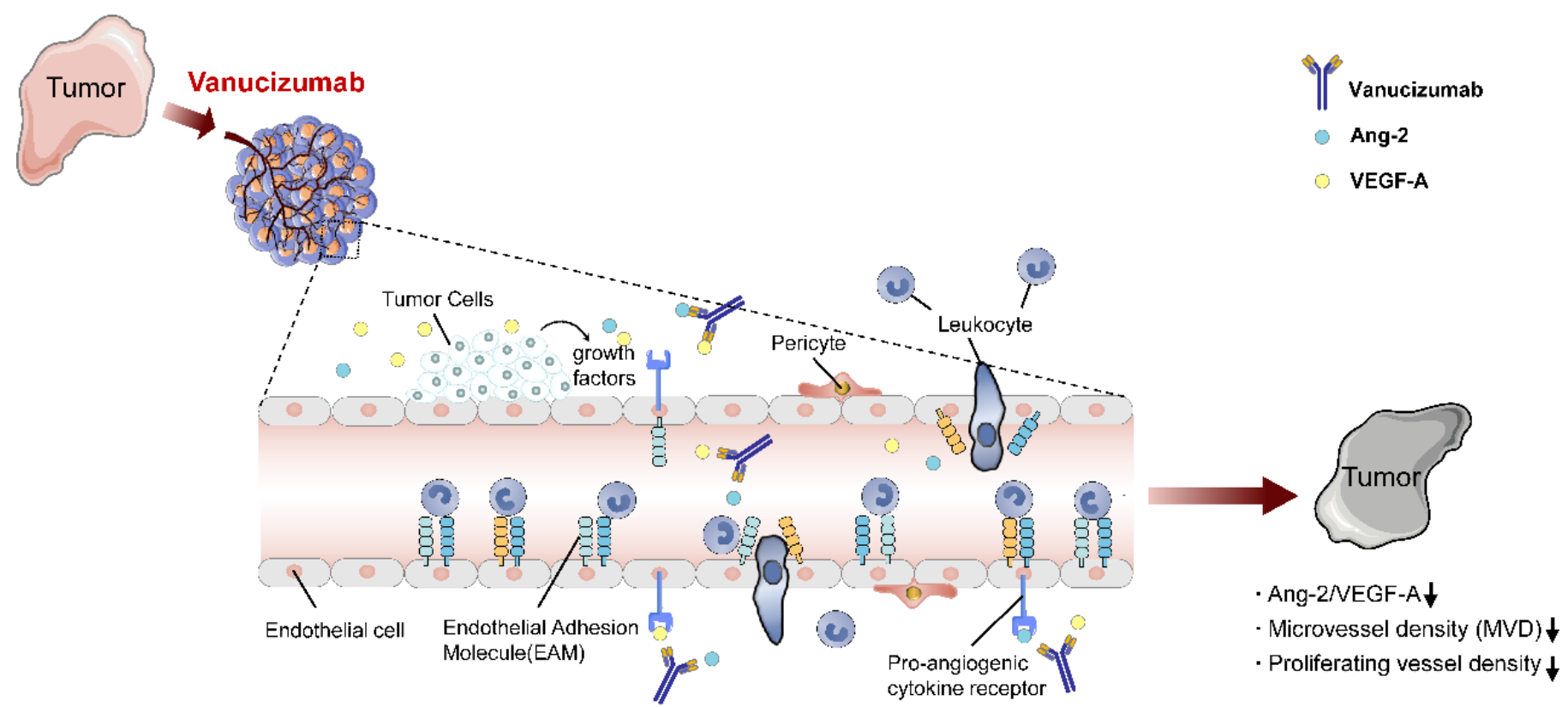

Figure 1 Biological effects of vanucizumab treatment on advanced solid tumors.

Vanucizumab specifically binds to VEGF and Ang-2 antigens to block their biological activities, then decreases vascular-related biomarkers VEGF-A and Ang-2 in the plasma, microvessel density (MVD), and density of proliferating vessels in tumor and skin-wound-healing biopsies. 